\title{
Loneliness Among Older People: Results from the Swedish National Study on Aging and Care - Blekinge
}

\author{
Elin Taube ${ }^{*}, 1,2,3,4$, Jimmie Kristensson $^{4}$, Patrik Midlöv ${ }^{1,2}$, Göran Holst ${ }^{5}$ and Ulf Jakobsson ${ }^{1,2}$ \\ ${ }^{I}$ Center for Primary Health Care Research, Faculty of Medicine, Lund University, Malmö, Sweden \\ ${ }^{2}$ Department of Clinical Sciences in Malmö, Faculty of Medicine, Lund University, Malmö, Sweden \\ ${ }^{3}$ The Vårdal Institute, the Swedish Institute for Health Sciences, Lund University, Lund, Sweden \\ ${ }^{4}$ Department of Health Sciences, Faculty of Medicine, Lund University, Lund, Sweden \\ ${ }^{5}$ School of Health Sciences, Blekinge Institute of Technology, Karlskrona, Sweden
}

\begin{abstract}
Objectives: To investigate the prevalence and predictors of loneliness in older people (aged $78+$ ) over a sixyear period.

Method: The sample ( $\mathrm{n}=828$ ) was drawn from the Swedish National Study on Aging and Care and the respondents were followed up at three and six years. Data were collected by means of structural interviews with supplementary questionnaires.

Results: Half of the respondents reported that they felt lonely sometimes or more often. Women, widows/-ers living alone were more prone to report loneliness. Both independent associated factors and predictors were identified showing that loneliness is associated with and predicted by both physical and psychosocial outcomes.

Discussion: Loneliness is common among older people and seems to be a steady state affected mainly by psychological and psychosocial factors such as personality, satisfaction with life, risk of depression, lack of friends and loss of spouse. Psychosocial interventions targeting emotional loneliness and social isolation are suggested.
\end{abstract}

Keywords: Aged, health, loneliness, predictors, psychosocial aspects.

\section{INTRODUCTION}

Loneliness is a phenomenon that occurs in all stages of life and is a significant problem for many older people. Previous research has shown that loneliness in old age is a risk factor that can be linked to various health-related problems, physical and mental problems [1-2]. Aging, in particular among the oldest $(80+)$ is accompanied by physical problems such as frailty and functional decline and mental problems such as reduced cognitive capacity and greater loneliness [3]. In Sweden, as well as in many other European countries, the population of older people is growing and over the coming decade the increase will be mainly accounted for by the oldest age groups (80+) [4-6]. Thus there will be a greater need for personal care and support as advanced old age is associated with disability $[4,6]$. Much research has focused on physical health problems among the oldest people but research into mental health and loneliness are to some extent still sparse.

The prevalence of loneliness in the aged population $(65+)$ varies among different study results and is dependent on the definition and the intensity of the feeling. A review by

*Address correspondence to this author at the Department of Health Sciences, Faculty of Medicine, Lund University, P.O Box 157, SE-221 00 Lund, Sweden; Tel: +46 46222 1944; Fax: +46 46 2221934;

E-mail: elin.taube@med.lu.se
Dykstra [7] reports a prevalence of $20-30 \%$ of moderate or serious loneliness among older people aged 65-79 years. However, in the oldest age group (80+), 40-50\% report that they are often lonely. Since loneliness is a unique experience for every individual it can be hard to define [8]. In 1973 Weiss [9] introduced what is now a widely used definition suggesting that loneliness can be defined from two different aspects; emotional loneliness, which is a result of the loss or the absence of someone close, usually a partner, relative or friend, and social isolation, which is a consequence of the lack of a network of involvement with other people or groups, for example co-workers, neighbors or friends. Based on previous research the oldest age group appears to be particularly vulnerable due to the negative effects of the aging process and the higher prevalence of loneliness. Therefore, assessing loneliness among the oldest people and the problems linked with the phenomenon constitute a research area of importance in extending knowledge and the ability to intervene.

Research into loneliness among older people has mainly been based on cross-sectional samples and the findings show a variety of factors, modifiable or static, to be associated with greater loneliness e.g. reduced capacity in activities of daily living, ADL [10], lower health-related quality of life, HRQoL [10], less satisfaction with life [11], reduced cognitive capacity $[12]$ and personality traits $[12,13]$ among others. 
Longitudinal studies of loneliness among older people have identified various predictors, however, there are few such studies. In a study by Aartsen and Jylhä [14] that followed individuals over 28 years $(n=469,1979)$, losing a partner was identified as an independent predictor for loneliness; a result supported by earlier studies [15, 16]. Other variables have also been identified as predictors that contribute to loneliness such as deterioration in health [1516], mobility [16], greater comorbidity and more doctors' visits [17]. Having fewer social activities [14], a limited social network e.g. in form of less emotional support, and having to spend holidays alone have also proved to be independent predictors for developing loneliness [17] together with increased feelings of depressed mood, nervousness and uselessness [14]. Nervousness can also be seen as a personality trait since it is one of the descriptive characteristics of the personality factor neuroticism [18], thus the study by Aartsen \& Jylhä [14] indicates that personality can predict loneliness. It has also been stated that loneliness increases with age [17] and the increase is greatest for the oldest [15]. On the other hand, improvements regarding factors such as social activity, quality of life rating and an increased number of confidants have shown to have a positive effect on levels of loneliness along with less deterioration in health and moving from living alone to live with others [19].

Knowledge derived from studies targeting predictors, particularly modifiable, for loneliness among older people is useful in the preventive aspect of the caring process. It is therefore useful to broaden existing knowledge in terms of identifying possible predictors for loneliness since that currently available is limited, especially from a long-term perspective.

According to previous findings the oldest constitute a vulnerable group regarding loneliness and related factors. Loneliness, among other psychosocial aspects, could be seen as a component that prohibits successful aging and a good quality of life. Cohen-Mansfield et al. [17] highlight the importance of investigating populations, which are especially vulnerable to loneliness in order to understand the mechanisms underlying the development of the phenomenon. Targeting the oldest age groups and using a longitudinal approach provides a possibility to further clarify the complexity of the phenomenon of loneliness, which in turn will be useful in developing clinical interventions to optimize care for the oldest people. The aim of this study was to investigate the prevalence and predictors of loneliness in older people $\left(78^{+}\right)$over a six-year period.

\section{METHOD}

\section{Sample}

This study comprises a sample of 828 people aged 78 years or older and drawn from the Swedish National Study on Aging and Care (SNAC). SNAC is a national, longitudinal study which includes four research centers in Sweden [20]. The sample is drawn from one of these centers, namely a county in the region of Blekinge (SNAC-B). SNAC-B covers one municipality in the southeastern part of Sweden with approximately 60600 inhabitants, including urban and rural areas. The municipality/sample resembles other subpopulations in Sweden regarding age distribution, gender and functional ability. Data collection for the baseline survey was carried out from 2001-2003 and of those who were asked $61 \%$ agreed to participate $(\mathrm{n}=1402)$. The total sample, consisting of 585 men and 817 women, is divided in ten age cohorts $(60,66,72,78,81,84,87,90,93,96)$ ranging from 60 to 96 years of age. The two most common reasons among those 910 people who did not choose to participate were: did not want to $(83 \%)$ and being too ill $(10 \%)$. The response rate ranged between $55-75 \%$ and was lowest for the oldest people compared to the youngest people who had the highest. The sample in the present study includes individuals aged 78 years or older at baseline. Age cohorts of individuals aged 78 years or older were followed-up every third year (2004-2006 \& 2007-2009). The two follow-ups, in 20042006 and in 2007-2009 respectively were included in this study. Thus, the sample in the present study is drawn from all the three measuring points and includes individuals aged 78 years or older at baseline. The final sample in the present study comprises 828 individuals at baseline (2001-2003), 511 individuals in the 2004-2006 follow-up and finally 317 individuals in the 2007-2009 follow-up. The study was ethically approved by the Regional Ethics Review Board of Lund (LU 650/00, LU 744/00).

\section{Data Collection}

An invitation to participate in the study and visit the research centre was mailed to potential participants at two occasions. Those who did not respond to the letter were invited again by telephone. During the first session informed consent was obtained from the participants. Data were collected at the research centre or in the respondents' homes by means of structural interviews and medical examinations with supplementary questionnaires. The research team included registered nurses or physicians. If it was needed the respondents were offered help completing the questionnaire. For those who could not complete the questionnaire, despite help, a special questionnaire regarding the respondent was given to a family member to fill out. This occurred in 30 cases, however not included in this study, out of a total of 1402 informants at baseline.

\section{Measurements}

Various measurements, scales and single-item questions were used in order to obtain data regarding a specific area or phenomenon. The measurements, scales and single-item questions have been previously used and validated in a Swedish context. Four single-item questions were used to measure loneliness by the experience, intensity, comparability with others of the same age and frequency. Social contacts were also measured on single-item questions concerning contact with their own children, having a sufficient number of friends, having a confidant and wanting more contact with friends, family and neighbors.

Levels of satisfaction with life were obtained by using the Life Satisfaction Index Z (LSIZ) [21] consisting 13 items in statement form, including both negative and positive statements, on a three-point Likert scale (agree, don't know, disagree). The score ranges from $0-26$ and a higher score indicates greater satisfaction. The LSIZ has been translated into Swedish and the instrument is suitable for measuring general life satisfaction in older people [22]. HRQoL was measured using EQ-5D [23], which covers five dimensions 
of health (mobility, self-care, usual activities, pain/discomfort, anxiety/depression) with three response levels (no problem, some problems, severe problems). A score is generated based on the five answers, yielding a utility score ranging from 0.00 (dead) to 1.00 (perfect health) [24].

The classification of personality domains was made using a Swedish version of the Neo-Five Factor Inventory (NEOFFI) [25] which has been used previously [26]. The instrument consists of 60 items describing five basic domains of personality (the Five Factor Model): neurotic, extraversion, openness, agreeableness and conscientiousness. The self-rating scale is based on statements, both negative and positive, on a 1-to-5 scale (strongly disagree to strongly agree) and summed up to yield five domain scores. Low and high scores from the domains can then be used to characterize the respondent.

In order to assess cognitive capacity the Mini Mental State Exam (MMSE) was used. The instrument captures the cognitive aspects of mental functions, comprised in eleven items yielding a total score of 30 points, where a low score indicates lower cognitive capacity [27].

Self-reported health complaints were measured by means of eleven different complaints/items. The complaint should have troubled the respondent during the last three months and was answered with yes or no. The original version comprises 30 different complaints covering physiological and psychological functions [28].

Activities of daily living (ADL) were assessed using questions that directly corresponded to the ADL staircase [29]. The ADL staircase assesses dependence/independence in daily living and comprises six personal activities of daily life (PADL) - bathing, dressing, going to the toilet, transfer, continence, feeding and four instrumental activities of daily living (IADL) - cleaning, shopping, transportation and cooking. The response alternatives were dichotomized (can or cannot) with a maximum total score of 10 . Respondents with a score of 0 were defined as independent and 1-10 as dependent in ADL.

\section{Data Analysis}

Comparisons were made between those individuals who reported loneliness and not. Comparisons were made between the two groups and a set of variables chosen according to relevance and previous knowledge. Loneliness was the dependent variable throughout the analyses and dichotomized as not lonely (0) and lonely (1) based on the question "Do you ever feel lonely?" with four response alternatives. The lonely group (1) includes those individuals who answered "sometimes" or "often" and the not lonely group (0) includes those who answered "seldom" or "never". In this study Student's $t$-test was used for normally distributed interval/ratio data, the Mann-Whitney $U$-test for ordinal and interval/ratio data which were not normally distributed and the Chi-square test or Fisher's Exact test for nominal data. For repeated measures the Friedman test was used and for the post-hoc analysis Wilcoxon's Signed Rank test was used. In addition, a reduced $p$-value, Bonferroni correction method, was used to control for Type 1 error [30] in the post-hoc analysis. To identify possible predictors and associated factors for loneliness multiple logistic regression analysis (backward, manual) was performed. The Hosmer and Lemeshow goodness-of-fit test and Nagelkerke $R$-square [31] served as tests for the goodness-of-fit for the regression models. Three regression models were made, one for each measuring point (2001, 2004, 2007), including following independent variables (baseline data); gender, age, marital status, living alone, ADL-scale sum, personality traits (neurotic, extroversion, openness, agreeableness, conscientiousness), HRQoL, life satisfaction, health complaints (depressed mood, fatigue, leg pain, hearing loss), cognitive capacity and lacking friends. In addition to this, being lonely at baseline was entered in to the models for 2004 and 2007. In all statistical analyses a $p$-value $\leq 0.05$ was considered as significant, except for post-hoc analyses where a reduced $p$-value was used, according to the Bonferroni correction method. All data were analyzed by using PASW Statistics 18.0 .

\section{RESULTS}

At baseline (2001) the mean age of the sample was 84.2 years (78-96 years, SD 4.7) at the follow-up in 200483.8 years (78-99 years, SD 4.3) and at the 2007 follow-up 84.1 years (78-99 years, SD 4.6). At baseline $59 \%$ were women, over $60 \%$ were living alone and $53 \%$ were widows/-ers (Table 1). The majority $(90.0 \%)$ lived in ordinary housing such as an apartment, house etc. (Table 1). When comparing those individuals who felt lonely at baseline with those who did not significantly more of the former were women $(71 \%)$, a widow/-er $(67 \%)$, living in residential care $(11 \%)$ or living alone $(80 \%)$.

Both the prevalence and the intensity of the feeling of loneliness at the three measuring points (2001, 2004 and 2007) showed that around $50 \%$ of the participants felt lonely at least sometimes or even more often and the intensity level was described as "neither strong nor weak" by around $55 \%$ (Table 2).

\section{Loneliness in Relation to Other Variables, Cross- Sectional Comparisons}

The participants who were lonely scored significantly lower in the LSIZ (life satisfaction) at all measuring points (Table 3). Furthermore, the LSIZ score decreased over time for both groups, but a slightly greater decrease was found among those who were lonely (Table 3). Regarding health related quality of life, a significant difference in the EQ5D score could be seen at all measuring points with those who were lonely scoring lower (Table 3). Differences were also found when comparing cognitive capacity between those who were lonely and those who were not in that lonely participants scored significantly lower on the MMSE in 2001. However, for the remaining years the differences were small and not significant (Table 3). When comparing the ability to perform activities of daily living between the two groups, the score for the ADL staircase showed significant differences at all points (Table 3 ). Those who were lonely scored higher at all measuring points, indicating a reduced capacity to perform ADL (Table $\mathbf{3}$ ). 
Table 1. Sociodemographic Variables at Baseline (2001) Including a Comparison Between Respondents Reporting Loneliness and Not

\begin{tabular}{|c|c|c|c|c|}
\hline & Total Sample $(\mathrm{n}=\mathbf{8 2 8})$ & Not Lonely* $(n=347)$ & Lonely* $(n=371)$ & $p$-Value \\
\hline Age mean (SD) & $84.2^{\mathrm{a}}(4.68)$ & $83.0(4.18)$ & $84.6(4.62)$ & $<0.001^{\mathrm{t}}$ \\
\hline Female & 59.4 & 48.1 & 71.2 & \\
\hline Male & 40.6 & 51.9 & 28.8 & \\
\hline Married & 34.9 & 53.5 & 19.2 & \\
\hline Widow/widower & 52.9 & 36.6 & 67.4 & \\
\hline Unmarried & 7.8 & 6.7 & 7.9 & \\
\hline Divorced & 4.4 & 3.2 & 5.5 & \\
\hline$\geq 4$ & 17.5 & 17.1 & 18.0 & \\
\hline \multicolumn{4}{|l|}{ Living Arrangements (\%) } & 0.001 \\
\hline Ordinary housing & 90.0 & 95.9 & 88.8 & \\
\hline Residential care/sheltered housing & 10.0 & 4.1 & 11.2 & \\
\hline Living alone (\%) & 61.6 & 40.3 & 79.6 & $<0.001$ \\
\hline With spouse (\%) & 34.3 & 54.8 & 17.9 & $<0.001$ \\
\hline
\end{tabular}

The prevalence of perceived depressed mood as a health complaint differed significantly between the two groups in 2001 and 2007, with the lonely presenting the highest prevalence (Table 4). A similar pattern can be seen in perceived nervousness, as a health complaint, where the lonely participants reported a significantly higher prevalence in 2001 and 2004 (Table 4). Regarding the other perceived health complaints (Table 4) lonely participants had a higher prevalence for all complaints at all measuring points, apart from two complaints in 2007 (hearing loss and backache). Taking all three points into account the most common complaints for both groups and the total sample were fatigue, hearing loss and leg pain (Table 4).

Over $20 \%$ of those who were lonely did not have enough friends compared to those who were not lonely, were approximately $5 \%$ reported that they lacked friends, the difference between the groups was significant and all three measuring points was taken into account (Table 5). In addition, over $40 \%$ of those who were lonely wanted more contact with family, friends and neighbors compared to those who were not lonely where around $21 \%$ wanted more contact, again the difference was significant (Table 5). However, over $90 \%$ of the participants in both groups and at all measuring points had a confidant, with no significant differences found between the groups (Table 5).
Personality, according to the FFM, is based on five different personality traits: neurotic, extroversion, openness, agreeableness and conscientiousness. A comparison was made between the two groups at baseline and significant differences could be found in four of the five traits when using a modified version of the NEO-FFI (Table 6). The lonely scored higher for neurotic and extroversion and lower for openness and conscientiousness, compared to those participants who were not lonely (Table 6).

\section{Predictors of Loneliness}

A set of variables at baseline was used in a logistic regression model in order to find predictors of loneliness in 2004 and 2007. For 2001 the model generates independent variables, which can be seen as factors associated with loneliness instead of predictors. The same set of variables was used in all three models with an addition of one variable, "lonely at baseline", in 2004 and 2007.

In 2001, at baseline, the analysis resulted in seven independent, associated factors for loneliness: living alone, lacking friends and depressed mood as a health complaint. Two personality traits were associated with loneliness: neurotic and conscientiousness (Table 7). The five independent variables indicate a probability of an increase in loneliness. The remaining two, life satisfaction and HRQoL 
Table 2. Prevalence of Loneliness at Baseline and Follow-Ups, Including a Comparison Between the Measuring Points

\begin{tabular}{|c|c|c|c|c|c|}
\hline & $2001(n=828)$ & $2004(n=511)$ & $2007(n=317)$ & $p$-Value & Post-Hoc Analyses \\
\hline \multicolumn{4}{|c|}{ Do You Ever Feel Lonely? (\%) } & $0.005^{\mathrm{a}}$ & $\mathrm{A}, \mathrm{B}^{\mathrm{b}},-$ \\
\hline Often & 8.8 & 7.5 & 8.8 & & \\
\hline Sometimes & 42.9 & 43.5 & 40.6 & & \\
\hline Seldom & 29.0 & 32.3 & 31.8 & & \\
\hline Never & 19.4 & 16.7 & 18.8 & & \\
\hline \multicolumn{4}{|c|}{ When You Feel Lonely, How Strong is Your Feeling of Loneliness? (\%) } & $0.022^{\mathrm{a}}$ & $\mathrm{A}, \mathrm{B}^{\mathrm{b}},-$ \\
\hline Very strong & 4.1 & 5.7 & 3.6 & & \\
\hline Rather strong & 18.3 & 23.6 & 17.1 & & \\
\hline Neither nor & 49.1 & 54.2 & 60.7 & & \\
\hline Rather weak & 17.8 & 12.7 & 15.7 & & \\
\hline Very weak & 10.6 & 3.8 & 2.9 & & \\
\hline \multicolumn{4}{|c|}{ Compared to Others of Your Age, How Lonely are You? (\%) } & $0.299^{\mathrm{a}}$ & - \\
\hline Much more & 2.7 & 2.4 & 3.4 & & \\
\hline A little bit more & 8.0 & 7.0 & 8.5 & & \\
\hline The same & 34.3 & 33.1 & 31.6 & & \\
\hline Less & 27.9 & 31.0 & 27.8 & & \\
\hline Much less & 27.1 & 26.4 & 28.6 & & \\
\hline \multicolumn{4}{|c|}{ When You Look Back at the Last Five Years, which Alternative Fits You?* (\%) } & $0.120^{\mathrm{a}}$ & - \\
\hline No occasions & 34.9 & 41.5 & 35.6 & & \\
\hline Occasional & 50.9 & 43.6 & 48.3 & & \\
\hline Recurring Occasions & 11.1 & 11.6 & 12.7 & & \\
\hline Continuous & 3.0 & 3.3 & 3.4 & & \\
\hline
\end{tabular}

*No occasions with feelings of loneliness; Occasional feelings of loneliness; Recurring occasions of loneliness; Continuous feelings of loneliness.

Missing: $13.3 \%-32.9 \%$ (2001), 33.3\%-59.7\% (2004), 24.9\%-56.2\% (2007).

Significant differences between: (A) 2001 vs 2004, (B) 2001 vs 2007, (C) 2004 vs 2007.

a: Friedman test.

b: Wilcoxon's Signed Rank test.

Reduced $p$-value (Bonferroni) for post-hoc analyses: $<0.0167$.

Table 3. Comparison at Baseline and Follow-Ups Between Respondents Reporting Loneliness and Not Regarding Life Satisfaction, Health Related Quality of Life, Cognitive Capacity and Activities in Daily Living

\begin{tabular}{|c|c|c|c|c|c|c|c|c|c|}
\hline & \multicolumn{3}{|c|}{$2001(n=828)$} & \multicolumn{3}{|c|}{$2004(n=517)$} & \multicolumn{3}{|c|}{$2007(n=318)$} \\
\hline HRQoL, EQ5D mean (SD) & $0.78(0.21)$ & $0.64(0.27)$ & $<\mathbf{0 . 0 0 1}^{\mathrm{b}}$ & $0.78(0.21)$ & $0.65(0.25)$ & $<0.001^{\text {b }}$ & $0.75(0.20)$ & $0.66(0.27)$ & $0.005^{b}$ \\
\hline Cognitive Capacity, MMSE mean (SD) & $25.91(3.85)$ & $24.32(5.18)$ & $<\mathbf{0 . 0 0 1}^{\mathrm{b}}$ & $26.45(2.84)$ & $25.74(3.61)$ & $0.255^{\mathrm{b}}$ & $25.88(3.40)$ & $26.03(2.79)$ & $0.841^{\mathrm{b}}$ \\
\hline
\end{tabular}

a: Student's $t$-test.

b: Mann-Whitney $U$-test.

Missing: 14.7\%-29.2\% (2001), 37.0\%-55.6\% (2004), 25.2\%-29.3\% (2007)

* "Do you ever feel lonely?" Not Lonely (0): "seldom" or "never" Lonely (1): "sometimes" or "often".

were also identified and lower scores on the LSIZ and the EQ5D indicate a higher probability of being lonely (Table 7 ). Altogether, the seven variables explained $45 \%$ of the total variance in loneliness at baseline (Table 7).
Four independent variables predicting loneliness over time were identified in the model for 2004 and together the variables explained $43 \%$ of the total variance in loneliness (Table 7). Lonely at baseline, suffering from leg pain, living 
Table 4. Comparison at Baseline and Follow-Ups Between Participants Reporting Loneliness or Not Regarding Various Health Complaints During the Last Three Months, or Longer

\begin{tabular}{|c|c|c|c|c|c|c|c|c|c|c|c|c|}
\hline & \multicolumn{4}{|c|}{$2001(n=828)$} & \multicolumn{4}{|c|}{$2004(n=517)$} & \multicolumn{4}{|c|}{$2007(n=318)$} \\
\hline Nervous (\%) & 19.0 & 11.2 & 26.5 & $<0.001$ & 15.7 & 9.0 & 22.1 & 0.001 & 18.1 & 14.0 & 22.2 & 0.101 \\
\hline Fatigue (\%) & 52.8 & 44.7 & 60.3 & $<0.001$ & 61.6 & 52.2 & 70.6 & 0.001 & 58.0 & 51.2 & 65.0 & 0.032 \\
\hline Walking difficulties (\%) & 43.3 & 35.1 & 51.0 & $<0.001$ & 45.0 & 36.9 & 52.7 & 0.004 & 48.3 & 45.5 & 51.3 & 0.368 \\
\hline Dizziness (\%) & 30.0 & 23.6 & 36.1 & $<0.001$ & 32.7 & 26.9 & 38.3 & 0.031 & 36.1 & 34.7 & 37.6 & 0.642 \\
\hline Hearing loss $(\%)$ & 54.4 & 49.4 & 59.2 & 0.010 & 58.5 & 57.6 & 59.4 & 0.743 & 64.3 & 64.5 & 64.1 & 0.954 \\
\hline Stomach pain $(\%)$ & 20.1 & 14.7 & 25.2 & 0.001 & 21.6 & 16.8 & 26.2 & 0.041 & 17.2 & 10.7 & 23.9 & 0.007 \\
\hline
\end{tabular}

a: Pearson's Chi' ${ }^{2}$-test.

Missing: $15.1 \%-19.8 \%$ (2001), 38.0\%-39.0\% (2004), 25.2\% (2007).

* "Do you ever feel lonely?" Not Lonely (0): "seldom" or "never" Lonely (1): "sometimes" or "often".

Table 5. Comparison at Baseline and Follow-Ups Between Participants Reporting Loneliness or Not Regarding Aspects of Social Network and Living Arrangements

\begin{tabular}{|c|c|c|c|c|c|c|c|c|c|c|c|c|}
\hline & \multicolumn{4}{|c|}{$2001(n=828)$} & \multicolumn{4}{|c|}{$2004(n=517)$} & \multicolumn{4}{|c|}{$2007(n=318)$} \\
\hline & $\begin{array}{c}\text { Total } \\
\text { Sample }\end{array}$ & $\begin{array}{c}\text { Not } \\
\text { Lonely* }\end{array}$ & Lonely* & $\begin{array}{c}p \text { - } \\
\text { Value }\end{array}$ & $\begin{array}{c}\text { Total } \\
\text { Sample }\end{array}$ & $\begin{array}{c}\text { Not } \\
\text { Lonely* }\end{array}$ & Lonely* & $\begin{array}{c}p- \\
\text { Value }\end{array}$ & $\begin{array}{c}\text { Total } \\
\text { Sample }\end{array}$ & $\begin{array}{c}\text { Not } \\
\text { Lonely* }\end{array}$ & Lonely* & $\begin{array}{c}p- \\
\text { Value }\end{array}$ \\
\hline Children ${ }^{1}(\%)$ & 86.4 & 92.5 & 80.9 & $<0.001^{a}$ & 88.4 & 91.5 & 85.2 & $0.096^{\mathrm{a}}$ & 90.8 & 96.1 & 85.1 & $0.008^{\mathrm{a}}$ \\
\hline Friends ${ }^{2}(\%)$ & 87.2 & 95.3 & 79.6 & $<0.001^{a}$ & 84.5 & 93.4 & 76.0 & $<0.001^{a}$ & 86.0 & 96.6 & 75.2 & $<\mathbf{0 . 0 0 1}^{\mathrm{a}}$ \\
\hline Confidant $^{3}(\%)$ & 96.0 & 96.2 & 95.9 & $0.852^{\mathrm{a}}$ & 95.0 & 97.0 & 93.1 & $0.108^{\mathrm{a}}$ & 93.7 & 95.8 & 91.5 & $0.166^{\mathrm{a}}$ \\
\hline Wanting more contact ${ }^{4}(\%)$ & No values** & & & & 32.6 & 20.8 & 44.2 & $<0.001^{a}$ & 32.6 & 22.0 & 43.5 & $<0.001^{\mathrm{a}}$ \\
\hline Form of housing (\%) & & & & $<0.001^{a}$ & & & & $0.030^{b}$ & & & & $0.007^{\mathrm{a}}$ \\
\hline Ordinary & & 95.9 & 88.8 & & & 100.0 & 96.5 & & & 96.6 & 87.0 & \\
\hline Residential Care & & 4.1 & 11.2 & & & 0.0 & 3.5 & & & 3.4 & 13.0 & \\
\hline Satisfaction with housing (\%) & & 93.8 & 95.7 & $0.208^{\mathrm{a}}$ & & 97.0 & 95.8 & $0.542^{\mathrm{a}}$ & & 96.6 & 93.7 & $0.302^{\mathrm{a}}$ \\
\hline
\end{tabular}

a: Pearson's $\mathrm{Chi}^{2}$ - Test.

b: Fisher's Exact Test.

1: "Do you consider the contact with your children being sufficient?" (yes).

2: "Do you consider your number of friends being sufficient?" (yes).

3: "Do you have someone who you feel that you can be yourself in front of and who accepts you with all your advantages and shortcomings?" (yes).

4: "Would you like to have more contact with relatives, friends and neighbors?" (yes).

Missing: 14.7\%-28.7\% (2001), 34.4\%-45.6\% (2004), 25.6\%-38.5\% (2007).

* Do you ever feel lonely? " Not Lonely (0): "seldom" or “never" Lonely (1): "sometimes" or "often".

** The question was not included at baseline.

alone were identified along with life satisfaction, which indicates that a lower score on the LSIZ increases the probability of developing loneliness over time (Table 7).

In the model for 2007 three independent predictors were identified: being lonely at baseline turned out to be a predictor for developing loneliness over time along with increased age and the personality trait openness which indicates that people who tend to be less open in their personality have an increased probability of developing loneliness over time (Table 7). The three predictors in the model for 2007 explain $24 \%$ of the total variance in loneliness (Table 7).

\section{DISCUSSION}

The findings from this study indicate that older people $(78+)$ who are lonely have less satisfaction with life, lower HRQoL, higher prevalence in subjective health complaints, lower capacity to perform ADL and a greater desire for increased social contacts, compared with those who are not 
lonely (Tables 3-5). Longitudinal findings show that both physical and psychological variables predict loneliness and also, once established, the experience of loneliness is likely to be permanent. Based on the findings it can be concluded that loneliness is linked to both physical and psychosocial factors, over time as well currently.

Table 6. Personality Domains at Baseline Based on the FiveFactor Model (NEO-FFI), a Comparison Between Participants Reporting Loneliness or Not

\begin{tabular}{|l|c|c|c|}
\hline Personality (NEO-FFI) & Not Lonely* & Lonely* & $\boldsymbol{p}$-Value \\
\hline \hline Neuroticism mean (SD) & $26.09(6.74)$ & $30.84(6.35)$ & $<\mathbf{0 . 0 0 1}$ \\
\hline Extroversion mean (SD) & $38.78(6.18)$ & $36.82(5.64)$ & $<\mathbf{0 . 0 0 1}$ \\
\hline Openness mean (SD) & $35.35(4.90)$ & $34.35(4.90)$ & $\mathbf{0 . 0 1 3}$ \\
\hline Agreeableness mean (SD) & $46.01(4.26)$ & $45.73(4.61)$ & 0.449 \\
\hline Conscientiousness mean (SD) & $44.99(5.97)$ & $43.72(5.52)$ & $\mathbf{0 . 0 1 0}$ \\
\hline
\end{tabular}

a: Student's $t$-test.

Missing: 32.0\%-35.4\%.

* Do you ever feel lonely?" Not Lonely (0):"seldom" or "never" Lonely (1): "sometimes" or "often".

The result indicates that loneliness is a common problem among older people, as many as $50 \%$ of the participants felt lonely sometimes or often (Table 2). This is in line with previous research reporting that $40-50 \%$ of people aged 80 and above are often lonely [7]. Moreover, feelings of loneliness have been shown to be more prevalent in the oldest age groups $(80+)$ compared to the younger [32, 33]. However, the prevalence does not explain the experience of loneliness and its consequences for health and health-related outcomes. Loneliness is known to have negative impacts in terms of both physical and mental health, such as higher blood pressure [2], poor self-rated health [34] and depressed mood [35] among others. On the other hand loneliness may not necessarily imply a negative experience; it can provide time for reflection and be self-fulfilling. This type of loneliness can be called solitude and is distinguished from the concept of loneliness [2]. Even if loneliness as an unwanted state seems to be common among older people it is not solely a negative experience for everyone, an aspect to consider when assessing loneliness in clinical practice.

This study showed that women, widows/-ers and those living alone were more likely to be lonely at baseline (Table 1) and this may be one explanation behind the rest of the results. It is well known that women live longer than men [36] and as a consequence both widowhood and thus living alone are more common among women. Loss of a spouse has been pointed out as a cause of loneliness in several studies $[10,14,37]$. Gender and marital status were, however, not identified as predictors of loneliness in this study (Table 7). Being a widow/-er may on the other hand be associated with living alone and since living alone often leads to a single-person household it could be assumed that

Table 7. Associated Variables and Predictors for Loneliness at Baseline and follow-Ups

\begin{tabular}{|c|c|c|c|c|c|c|}
\hline Final Model & $\mathbf{O R}^{\mathbf{a}, \mathbf{b}}$ & $95 \%$ CI for OR & $p$-Value & Unadjusted OR & 95\% CI for Unadjusted OR & $p$-Value \\
\hline \multicolumn{7}{|l|}{ Baseline, $2001(n=444)$} \\
\hline Lacking Friends & 4.260 & $1.833-9.928$ & 0.001 & 5.235 & $2.981-9.194$ & $<0.001$ \\
\hline Depressed Mood (Health Complaint) & 3.871 & $1.543-9.710$ & 0.004 & 3.471 & $2.165-5.564$ & $<0.001$ \\
\hline Consciensness & 1.063 & $1.012-1.115$ & 0.014 & 0.962 & $0.934-0.991$ & $<0.05$ \\
\hline Life Satisfaction (LSIZ) & 0.922 & $0.867-0.981$ & 0.011 & 0.828 & $0.794-0.864$ & $<0.001$ \\
\hline HRQoL (EQ5D) & 0.142 & $0.032-0.634$ & 0.011 & 0.074 & $0.033-0.165$ & $<0.001$ \\
\hline \multicolumn{7}{|l|}{ Follow-Up, $2004(n=298)$} \\
\hline Life Satisfaction (LSIZ) & 0.906 & $0.845-0.972$ & 0.006 & 0.848 & $0.799-0.900$ & $<0.001$ \\
\hline \multicolumn{7}{|l|}{ Follow-up, $2007(n=191)$} \\
\hline Lonely at Baseline & 5.415 & $2.782-10.541$ & $<0.001$ & 5.824 & $3.266-10.386$ & $<0.001$ \\
\hline Age & 1.137 & $1.020-1.269$ & 0.021 & 1.124 & $1.034-1.222$ & 0.006 \\
\hline Openness & 0.915 & $1.851-0.983$ & 0.015 & 0.918 & $1.859-0.980$ & 0.011 \\
\hline \multicolumn{7}{|c|}{$\begin{array}{l}\text { a: Nagelkerke } R^{2}: 0.453 \text { (2001), } 0.434 \text { (2004), } 0.237(2007) \\
\text { b: Hosmer and Lemeshow goodness-of-fit test: } 0.919 \text { (2001), } 0.919 \text { (2004), } 0.284 \text { (2007) } \\
\text { Missing values: } 46.4 \% \text { (2001), } 41.7 \% \text { (2004), } 39,7 \% \text { (2007) } \\
\text { Dependent variable dichotomized as: Do you ever feel lonely? "Not Lonely (0): "seldom" or “never" Lonely (1): "sometimes" or "often" } \\
\text { Variables included in the model: gender, age, marital status, living alone, ADL-staircase sum, personality (neurotic, extroversion, openness, agreeableness, consciousness), HRQo } \\
\text { (EQ5D), life satisfaction (LSIZ), health complaints (depressed mood, fatigue, leg pain, hearing loss), cognitive capacity (MMSE), lacking friends. The variable lonely at baseline wa } \\
\text { also included in the models for } 2004 \text { and 2007. Gender was dichotomized as male (0) female (1). }\end{array}$} \\
\hline
\end{tabular}


maintenance of the social network is important. Social contacts could, however, be inhibited by possible factors related to age such as deteriorating health and loss of peers, and could therefore be an additional reason for why loneliness being more common among those living alone. Based on the result two aspects of loneliness can be seen; emotional loneliness, which is a result of the loss or absence of someone close and social isolation, which is a consequence of an insufficient social network e.g. friends or neighbors [9]. Loss of a spouse and living alone can both result in emotional loneliness and social isolation can be a consequence of living alone. The findings from this study indicate that loneliness among older people should not be ignored; it is a prevalent and important aspect of a person's life situation and healthcare providers should make efforts to increase awareness of it as a potential problem and devise possible alleviating strategies.

Another result was that over $90 \%$ of the participants reported that they had a confidant i.e. someone to turn to and rely on, even among those who were lonely (Table 5). It is possible that the confidant represents the social dimension of loneliness, meaning that the aspects that relate to the emotional part are more difficult to realize in everyday life and perhaps even more so if the individual is widowed and/or lives alone. It is noteworthy that the question regarding a confidant was about whether the participant had one, not the quality of the relationship. However, the result indicates how complex the phenomenon of loneliness is and implies that more research is needed to achieve a deeper understanding, for instance through qualitative interview studies.

The analysis at baseline level identified the following independent factors associated with loneliness: living alone, depressed mood, lacking friends, lower HRQoL, lower life satisfaction and personality (neurotic and conscientiousness). All the factors mentioned have been shown to be associated with greater loneliness in previous research [2,10$11,13,35,38]$. Based on the results from this study the feeling of loneliness seems to be closely associated with the person's social network and psychological wellbeing. Psychological wellbeing may be affected by depressed mood, the individual's personality and satisfaction with life. It is interesting that psychosocial aspects play a more important role than physical aspects in relation to loneliness. Furthermore, this is important from a clinical point of view as the healthcare system is mainly focused on physical decline and disease and as a result risks failing to address loneliness before it becomes a health problem. A more holistic approach, including both physical and psychosocial interventions, is therefore needed in the care for older people.

The longitudinal results identified independent predictors for loneliness and the predictors differed depending on the time span (three $v s$ six yrs) however, loneliness at baseline was a strong predictor for experiencing loneliness both in the shorter and a longer term. This indicates that loneliness is a stable state and there is seemingly a risk that, once established, the feeling becomes permanent. Based on the findings here it is reasonable to assume that an old person suffering from loneliness will find it difficult to alleviate it by $\mathrm{him} /$ herself. This stresses the importance of making assessments to detect loneliness at an early stage and offering the person an intervention plan.

Since loneliness is such a complex phenomenon there are many possible factors co-variating, both on a psychosocial level and physical level. The associated variables and the predictors found in this study confirm that loneliness is affected by a broad spectrum of conditions related to both physical and psychosocial outcomes. For instance, suffering from leg pain or having a certain type of personality were both found to predict loneliness and this illustrates, to some extent, the complexity of the phenomenon. Suffering from leg pain could be limiting in itself but it could also be part of a broader situation encompassing deterioration in the overall health and multi morbidity, predictors that have been identified in earlier research [15-17]. Personality, or more exactly openness to experience, was found to predict loneliness and according to McCrae and Costa [39] personality brings order, predictability and continuity to the life course along with the ability to create or accommodate change. In conjunction with increased age changes might occur that affect the older person, such as the loss of a spouse and friends as well as a decline in overall health and so on. The finding that personality is a predictor implies that loneliness is dependent on how a person acts and handles the challenges that come with advanced age. Consequently, loneliness involves aspects on a multi-dimensional level from physical complaints to personality, however, this study has shown that the risk of being or becoming lonely mainly derives from psychosocial grounds rather than physical.

The main strength of this study was its longitudinal design, which, contributes to the existing body of knowledge regarding predictors for loneliness among older people. Furthermore, by looking at cross-sectional data and longitudinal data in the same study it was possible to achieve a more coherent picture. This is also useful in clinical practice since the findings provide knowledge about associated factors in order to identify those who are likely to feel lonely and predictors in order to identify those who are at risk of developing loneliness, or of continuing to feel lonely.

It is evident that loneliness is a common problem among the oldest people and, as highlighted by O'Luanaigh and Lawlor [2], both emotional loneliness and social isolation should be considered when planning for interventions. A possible approach to combating loneliness in older people could be to screen older persons at risk of being or developing loneliness and provide psychosocial interventions covering both aspects of loneliness as a regular part of the caring process.

\section{STUDY LIMITATIONS}

The study had some limitations which can be disussed in terms of threat to internal, external and construct validity [40]. A threat to internal validity was the attrition [40]. Between baseline (2001) and the last follow-up in 2007, 63 $\%(n=511)$ of the participants dropped out. When studying older people longitudinally it is inevitable that death or deterioration results in attrition. Therefore, it is possible that the sample in the follow-ups were healthier than the general population since it is reasonable to assume that the reasons for dropping out, beside death, was deteriorated health. This 
possible effect from the attrition is also a threat to external validity [41]. Efforts have been made in several ways in order to enroll and keeping the oldest in the study (e.g. by an over sampling of the oldest cohorts, create a friendly atmosphere, positive media contacts), however, the possible validity threats due to the attrition suggests that generalizations should be done with caution. Another possible threat to construct validity might be the use of single item questions when assessing loneliness. It assumes that the participants understands the concept of loneliness, a failure to explicate a construct may lead to incorrect inferences [40]. Allthough loneliness is a well known concept we can not rule out the possibility of misinterpretation. Also, it is possible that the experience was underreported since the questions do not take into account the fact that feeling lonely has negative connotations [32]. On the other hand, advantages that can be seen when assessing loneliness in form of single item questions are that it is easy to use in clinical and research settings, acceptable to people and approaches directly feelings of loneliness [2]. As previously mentioned, efforts have been made to provide a representative sample of the aging population and the sample size gave us adequate statistical power, which strengthens the statistical conclusion validity [40]. Finally, longitudinal studies concerning the oldest people are important and still quite rare. The design was a strength and allowed us to identify predictors for loneliness but again, we suggest that generalizations should be done with caution.

\section{CONCLUSION}

Loneliness is very common among the oldest people $(78+)$ and once a person becomes lonely he will probably continue to feel lonely. Factors associated with and predictors for loneliness have been shown to encompass both physical and psychological aspects on a multi-level. However, it seems that those factors which can be directly linked to psychological wellbeing are the major causes for older people being or becoming lonely. In clinical practice it is therefore important to recognize loneliness as a common issue, which needs to be targeted actively, mainly by means of psychosocial interventions covering emotional loneliness and social isolation, in order to prevent or alleviate it.

\section{CONFLICT OF INTEREST}

The authors confirm that this article content has no conflicts of interest.

\section{ACKNOWLEDGEMENTS}

The authors wish to thank all the respondents for participating in the main study. Furthermore, we would like to thank Pat Shrimpton for revising the English. The Swedish National Study on Aging and Care (www.snac.org) is financially supported by the Ministry of Health and Social Affairs, Sweden and the participating County Councils, Municipalities and University Departments. In addition, this study was supported by the Varrdal Institute, the Swedish Institute for Health Sciences, the Department of Health Sciences, Faculty of Medicine, Lund University and the Solstickan Foundation.

\section{REFERENCES}

[1] Donaldson JM, Watson R. Loneliness in elderly people: an important area for nursing research. J Adv Nurs 1996; 24: 952-9.

[2] O'Luanaigh C, Lawlor BA. Loneliness and the health of older people. Int J Geriatr Psychiatry 2008; 23: 1213-21.

[3] Baltes PB, Smith J. New frontiers in the future of successful aging: from successful aging of the young old to the dilemmas of the fourth age. Gerontology 2003; 49: 123-35.

[4] Lagergren M. Whither care of older persons in Sweden? - A prospective analysis based upon simulation model calculations, 2000-2030. Health Policy 2005; 74: 325-34.

[5] Persson G, Boström G, Allebeck P, et al. Chapter 5. Elderly people's health: 65 and after. Scand J Public Health Suppl 2001; 58: 117-31.

[6] Walker A, Maltby T. Ageing Europe. Buckingham: Open University Press.1997.

[7] Dykstra PA. Older adult loneliness: myths and realities. Eur J Ageing 2009; 6: 91-100.

[8] Killeen C. Loneliness: an epidemic in modern society. J Adv Nurs 1998; 28(4): 762-70.

[9] Weiss R. Loneliness: The experience of emotional and social isolation. Boston: The Massachusetts Institute of Technology. 1973

[10] Jakobsson U, Hallberg IR. Loneliness, fear and quality of life among elderly in Sweden: a gender perspective. Aging Clin Exp Res 2005; 17(6): 494-501.

[11] Borg C, Hallberg IR, Blomqvist K. Life satisfaction among older people $(65+)$ with reduced self-care capacity: The relationship to loneliness, overall health, physical activities and economic aspects. J Clin Nurs 2006; 15: 607-18.

[12] Martin, P, Hagberg B, Poon LW. Predictiors of loneliness in centenarians: A parallel study. J Cross Cult Gerontol 1997; 12: 203-24.

[13] Margrett JA, Daugherty K, Martin P, et al. Affect and loneliness among centenarians and the oldest old: The role of individual and social resources. Aging Ment Health 2011; 15(3): 385-96.

[14] Aartsen M, Jylhä M. Onset of loneliness in older adults: Results of a 28-year prospective study. Eur J Ageing 2011; 8: 31-8.

[15] Dykstra PA, Tilburg van TG, de Jong Gierveld J. Changes in older adult loneliness: Results from a seven-year longitudinal study. Res Aging 2005; 27(6): 725-47.

[16] Wenger CG, Burholt V. Changes in levels of social isolation and loneliness among older people in a rural area: A twenty-year longitudinal study. Can J Aging 2003; 23(2): 115-27.

[17] Cohen-Mansfield J, Shmotkin D, Goldberg S. Loneliness in old age: longitudinal changes and their determinants in an Israeli sample. Int Psychogeriatr 2009; 21(6): 1160-70.

[18] Pervin L. Personality: Theory and Research. New York: John Wiley \& Sons Inc., 2001.

[19] Victor CR, Bowling A. A longitudinal analysis of loneliness among older people in Great Britain. J Psychol 2012; 146(3): 313-31.

[20] Lagergren M, Fratiglioni L, Hallberg IR, et al. A longitudinal study intergrating population, care and social services data. The Swedish National study on Aging and Care (SNAC). Aging Clin Exp Res 2004; 16(2): 158-68.

[21] Wood V, Wylie ML, Sheafor B. An analysis of a short self-report measure of life satisfaction: Correlation with rater judgments. J Gerontol 1969; 24: 465-9.

[22] Fagerström C, Holst G, Hallberg IR. Feeling hindered by health problems and functional capacity at 60 years and above. Arch Gerontol Geriatr 2006; 44: 181-201.

[23] Brooks R. EuroQol: the current state of play. Health Policy 1996; 37: 53-72.

[24] Wolfs CAG, Dirksen CD, Kessels A, Willelms DCM, Verhey FRJ, Severens JL. Performance of the EQ-5D and the EQ-5D+C in elderly patients with cognitive impairments. Health Qual Life Outcomes 2007; 5: 33 .

[25] Costa, PT, McCrae, RR. Revised NEO personality inventory and NEO five- factor inventory professional manual. Odessa: Psychological Assessment Resources Inc. 1992.

[26] Rennemark M, Berggren T. Relationships between work-status and leisure lifestyle at 60 years of age. Eur J Ageing 2006; 3: 82-8. 
[27] Folstein MF, Folstein SE, McHugh PR. Mini Mental State: A practical method for grading the cognitive state of patients for the clinician. J Psychiatr Res 1975; 12: 189-98.

[28] Tibblin G, Bengtsson C, Furunes B, Lapidus L. Symptoms by age and sex: The population studies of men and women in Gothenburg, Sweden. Scand J Prim Health Care 1990; 8: 9-17.

[29] Åsberg HK, Sonn U. The cumulative structure of personal and instrumental ADL: A study of elderly people in a health service district. Scand J Rehabil Med 1989; 21: 171-7.

[30] Altman DG. Practical statistics for medical research. Boca Raton: Chapman and Hall/CRC 1991.

[31] Hosmer DW, Lemeshow S. Applied logistic regression. $2^{\text {nd }}$ ed., New York: Chichester 2000.

[32] Pinquart M, Sörensen S. Influences on loneliness in older adults: A meta-analysis. Basic Appl Soc Psych 2001; 23(4): 245-66.

[33] Savikko N, Routasalo P, Tilvis RS, Strandberg TE, Pitkälä KH. Predictors and subjective causes of loneliness in an aged population. Arch Gerontol Geriatr 2005; 41: 223-33.

[34] Nummela O, Seppänen M, Uutela, A. The effect of loneliness and change in loneliness on self-rated health (SRH): A longitudinal study among aging people. Arch Gerontol Geriatr 2011; 53: 163-7.
[35] Golden J, Conroy RM, Bruce I, et al. Loneliness, social support networks, mood and wellbeing in community-dwelling elderly. Int J Geriatr Psychiatry 2009; 24: 694-700.

[36] United Nations Statistics Division. Demographic Yearbook 20092010. Available from: http://unstats.un.org/unsd/demographic/prod ucts/dyb/dyb2009-2010.htm

[37] Victor CR, Scambler SJ, Bowling A, Bond J. The prevalence of, and risk factors for, loneliness in later life: A survey of older people in Great Britain. Ageing Soc 2005; 25: 357-75.

[38] Routasalo PE, Savikko N, Tilvis RS, Strandberg TE. Social contacts and their relationship to loneliness among aged people: A population based study. Gerontology 2006; 52: 181-7.

[39] McCrae RP, Costa PT. Personality in adulthood: A five-factor theory perspective. $2^{\text {nd }}$ ed. New York: The Guilford Press 2003.

[40] Shadish WR, Cook TD, Campell DT. Experimental and quasiexperimental designs for generalized causal inference. Boston: Houghton Mifflin Company 2002.

[41] Kazdin AE. Research design in clinical psychology. $4^{\text {ed }}$ ed. Boston: Allyn and Bacon Corporation 2003. 